\title{
Effect of off-axis cell orientation on mechanical properties in smooth muscle tissue
}

\author{
P. A. Sarma ${ }^{1,2}$, Ramana M. Pidaparti ${ }^{2,3}$, Richard A. Meiss ${ }^{4}$ \\ ${ }^{1}$ Department of Mathematics, Southern West Virginia Community and Technical College, Logan, USA; \\ ${ }^{2}$ Previously at the Department of Mechanical Engineering, Purdue School of Engineering and Technology, IUPUI, Indianapolis, \\ USA; \\ ${ }^{3}$ Department of Mechanical Engineering, Virginia Commonwealth University, Richmond, USA; \\ ${ }^{4}$ Department of OB/GYN, IU School of Medicine, Indiana University Medical Center, Indianapolis, USA. \\ Email:sarmap@southern.wvnet.edu, rmpidaparti@vcu.edu
}

Received August 18 2010; revised September 5 2010; accepted 9 September 2010.

\begin{abstract}
The cell alignment in a smooth muscle tissue plays a significant role in determining its mechanical properties. The off-axis cell orientation " $\theta$ " not only effects the shortening strain but also modifies the shear stress relationship significantly. Both experiments and finite element analysis were carried out on a tracheal smooth muscle strip to study how the cell alignment in smooth muscle affects the shear stiffness and shear stresses as well as deformation. A simple model for shear stiffness is derived using the data from experiments. Shear stiffness results obtained from the model indicate that the muscle shear stiffness values increase non-linearly with strain and with higher off-axis alignment of cells. Results of deformation and shear stresses obtained from finite element analysis indicate that the maximum shear stress values of tracheal smooth muscle tissue at $45 \%$ of strain are 2.5 times the corresponding values at $20 \%$ of strain for all three off-axis cell orientation values $\theta=$ $15^{\circ}, 30^{\circ}$ and $45^{\circ}$.
\end{abstract}

Keywords: Smooth Muscle; Shear Stress; Finite Element Model; Cell Orientation; Shortening Strain

\section{INTRODUCTION}

Smooth muscle tissue is an important part of any vascular system and the study of its mechanical properties has applications in organs like intestines, blood vessels, digestive tract, uterus and similar organs. Usually the smooth muscle tissue is arranged in circular and longitudinal layers that act antagonistically to shorten or lengthen and thus constrict or expand the volume of the organ. The muscle cells usually run parallel and are densely populated in an irregular manner. The mechani- cal properties of the tissue, such as force development, shortening and axial stiffness changes are due to the various cell activities within the tissue.

Specific architectural features of the tissue, such as the alignment of the cells along the major axis of the tissue strip, can also have significant effects on the mechanical properties. Several authors [1-4] investigated the axial stiffness of smooth muscle tissue and its variations with shortening length. Recently it has been shown that when the cells are not aligned along the major axis, the axial stiffness of the tissue is lower [5,6]. There have been no further studies in the literature reporting the shear stiffness for isolated smooth muscle tissues.

Meiss and Pidaparti $[5,6]$, in their study on the mechanical effects of off-axis cell orientation in a smooth muscle strip, have reported that the cell alignment in a smooth muscle tissue has a significant role in determining its length tension behavior and the isometric forces that it could generate. They further showed that the shortening capacity of the off-axis strip was greatly diminished, and the isometric force decreased much more rapidly with decreasing length. The effects of internal radial constraints based on tissue architecture were not significant at the lengths in question, nor could they explain the effects of off-axis cell orientation. Meiss and Pidaparti also noted that the shortening capacity and isometric force developed in the smooth muscle strip depended on its off-axis cellular orientation angle and that the relative stiffness increased with decreasing length much more than in the on-axis strip. This suggests that cells in these strips may have been free to move to a lower position on their intrinsic length-tension curves, although other possibilities, as reported here, were not ruled out.

It is usually difficult to conduct mechanical experiments on off-axis strips, especially in dissecting an iso- 
lated smooth muscle at a particular orientation. In this study, we investigate how the alignment of smooth muscle cells affects the shear stiffness of a tracheal smooth muscle strip through a simple model derived from experiments. In addition, the effects of off-axis cell orientation on shear stresses are investigated through a finite element analysis. The results of Von-mises stress and maximum shear stress values of the tissue are obtained and presented as functions of shortening strain and off-axis cell orientation $\theta$.

\section{MATERIALS AND METHODS}

\subsection{Experiments}

\subsubsection{Muscle Preparations}

All of the experiments were carried out on isolated strips of tracheal muscle from mongrel dogs. The animals were anaesthetised with pentobarbital sodium. Segments of 100 - to $150-\mathrm{mm}$-long extrathoracic trachea were quickly removed and placed in physiological saline solutions of the following millimolar compositions: $125 \mathrm{NaCl}, 4.7$
$\mathrm{KC1}, 2.5 \mathrm{CaCl}_{2}, 1.2 \mathrm{MgSO}_{4}, 15.5 \mathrm{NaHCO}_{3}, 1.2 \mathrm{KH}_{2} \mathrm{PO}_{4}$, and 11.5 glucose. The solutions were bubbled throughout the experiment with $95 \% \mathrm{O}_{2}$, and $5 \% \mathrm{CO}_{2}$ to maintain a physiological pH. As shown in Figure 1, the cartilaginous rings of the trachea were cut at both sides, and the preparation was pinned out in a dissecting dish. The muscle area was cleaned of epithelial and adventitial tissue. The muscle of the canine trachea consists of parallel bundles of muscle tissue with axially oriented cells. Small strips of muscle tissue $(\sim 0.75 \mathrm{~mm}$ diameter and $8-12 \mathrm{~mm}$ long) were cut from the muscle sheet, following the natural division of the tissue into discrete fiber bundles. These strips are termed 'on-axis'. To prepare 'off-axis strips', lengths of black thread were stretched across the tissue to mark the normal orientation and the desired off-axis angle ( $30^{\circ}$ in this case). The tissue was pinned to the dissecting dish along the thread guides so that it would not be distorted during the dissection. Using the angle thread as a guide, a strip similar to the control strip was dissected. To ensure a low-compliance

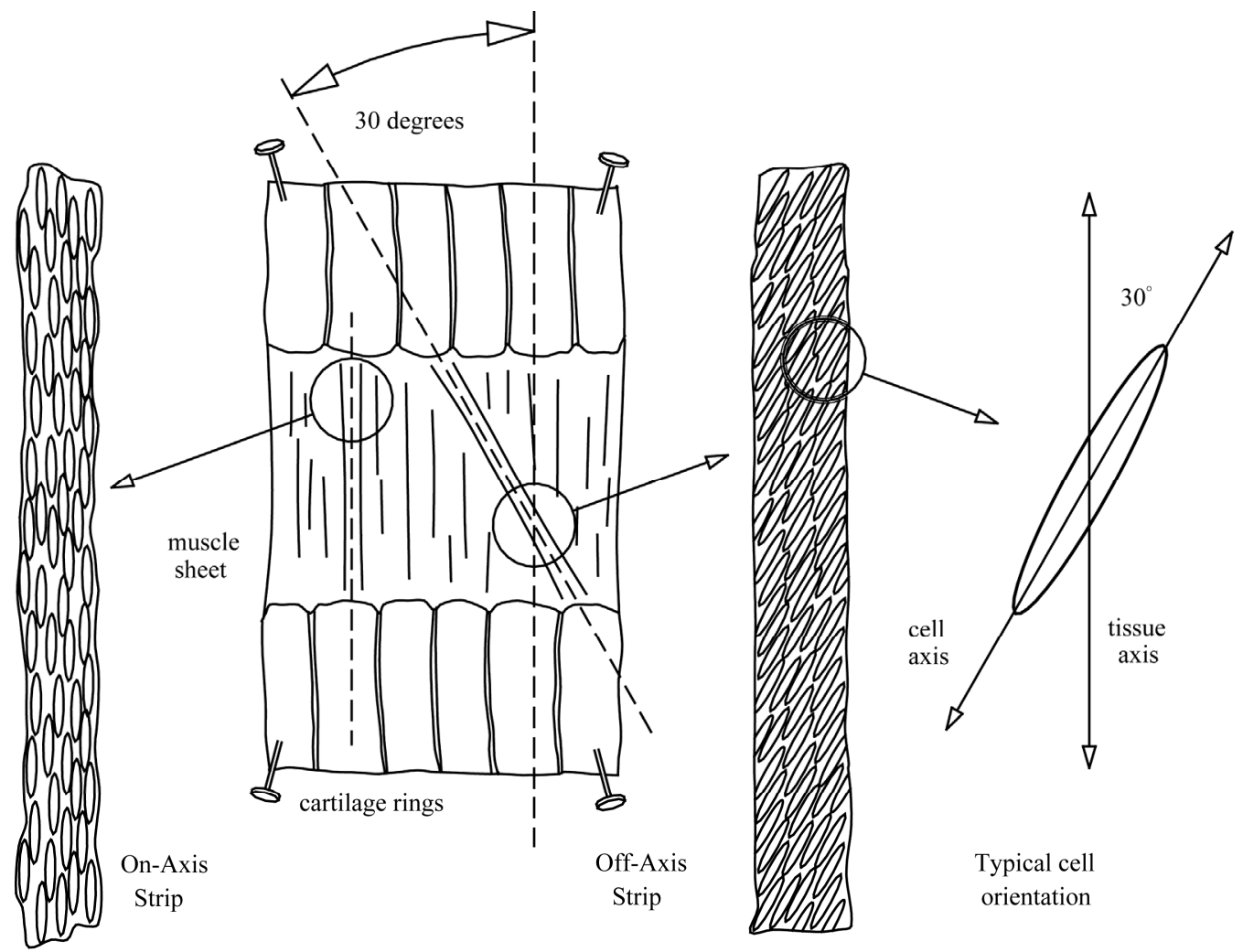

Figure 1. Dissection of off-axis tracheal muscle strips. A section of trachea approximately $30 \mathrm{~mm}$ long was removed from the excised organ. The cartilaginous rings were cut along both sides of the cylinder, and the posterior portion, containing the tracheal muscle sheet, was pinned down (as shown above) in a dissecting dish and stretched slightly to extend the fiber bundles. The On-Axis strip was cut from the muscle sheet parallel to the orientation of the fiber bundles. The Off-Axis strip was cut across the muscle sheet at a 30 degree angle to the axis of the fiber bundles. This produced a strip with cells oriented at 30 degrees with respect to the long axis of the strip. The typical orientation of a cell within the tissue is shown at the right. 
attachment to the experimental apparatus, the ends of all strips were clamped in aluminum foil cylinders as previously described [2]. Direct measurement of total system compliance, including that of the force transducer and all other mechanical components, gave a value of $0.93 \mu / \mathrm{mN}$, equivalent to $<0.5 \%$ of the muscle length at maximal force (e.g., $50 \mathrm{mN}$ ) at optimum length $\left(\mathrm{L}_{\mathrm{o}}\right)($ e.g., $10 \mathrm{~mm})$.

After mounting the tissue to the extension arms of the apparatus, it was extended by adjusting the position of the force transducer until a small force $(\sim 1-2 \%$ of the anticipated maximum) was recorded. This length was designated the rest length $\left(\mathrm{L}_{\mathrm{r}}\right)$ and was approximately $10 \%$ less than $L_{0}$. This procedure was followed because the experimental protocols required that passive force be kept to a minimum. After mounting the tissue, the muscle bath, borne on a rack-and-pinion assembly, was elevated to immerse the muscle in circulating, temperature-controlled, and oxygenated physiological saline solution. Muscles were stimulated by using platinum electrodes along either side of the tissue, with supramaximal voltage pulses of alternating polarity at a frequency and voltage previously determined to produce the maximum mechanical response.

\subsubsection{Mechanical Instrumentation}

All experimental contractions were made in a digitallycontrolled force-clamp servo system [2]. This system was capable of producing both isometric (length- controlled) and isotonic (force-controlled) conditions and of switching rapidly between them under manual or computer control. In addition, special conditions of length or force (i.e., length vibrations or rapid force steps) could be imposed. The continuous measurement of dynamic stiffness was performed as previously described, by applying a very small $\left(<0.5 \% \mathrm{~L}_{\mathrm{r}}\right)$ sinusoidal length oscillation (usually at $80 \mathrm{~Hz}$ ) to one end of the preparation and recording and analyzing the resulting force oscillation. These oscillations, superimposed on the length and force traces, were removed and quantified by a digitallycontrolled set of bandpass, notch, and low-pass filters. Force, length, and stiffness data were digitized and stored in computer memory for subsequent analysis.

\subsection{Shear Stiffness Model}

In this study, we developed a simple analytical model to predict the shear stiffness (resistance to off-axis loads or off-axis cells) of tracheal smooth muscle tissue as it is difficult to measure this stiffness experimentally. It was assumed that the off-axis smooth muscle tissue follows a similar behavior model to bone and other fiber-reinforced composites [7,8]. In addition, based on the architecture of the smooth muscle strip, it was further assumed that the tissue has negligible resistance in the transverse direction compared to the longitudinal direction of the strip. The off-axis stiffness coefficient $\left(\mathrm{E}_{\theta}\right)$ for the smooth muscle tissue was defined as

$$
\frac{1}{E_{\theta}}=\frac{\cos ^{4} \theta}{E_{x x}}+\left[\frac{1}{G_{x y}}-\frac{2 v_{x y}}{E_{x x}}\right] \cos ^{2} \theta \sin ^{2} \theta
$$

where $\theta$ is the angular orientation with respect to the long axis (on-axis) of the tissue strip, $\mathrm{E}_{x x}$ is the stiffness in the on-axis (axial) direction, $\mathrm{G}_{x y}$ is the shear stiffness, and $v_{x y}$ is the Poisson ratio. The shear stiffness, $\mathrm{G}_{x y}$ as a function of the shortening strain $\varepsilon$ can be estimated by knowing the on-axis stiffness $\left(\mathrm{E}_{x x}\right)$ and measuring the off-axis stiffness $\left(\mathrm{E}_{\theta}\right)$ for a particular orientation of the smooth muscle strip. A Poisson's ratio of 0.45 was assumed in the model, as there are no data available in the literature. Rearranging the Eq.1, the shear stiffness is given by

$$
\frac{1}{G_{x y}(\varepsilon, \theta)}=\left\{\frac{1}{E_{\theta}(\varepsilon, \theta)}-\frac{\cos ^{4} \theta}{E_{x x}(\varepsilon, \theta)}\right\} \frac{1}{\cos ^{2} \theta \sin ^{2} \theta}
$$

We used force-length experimental data for angular orientations $\theta=11^{\circ}$ and $30^{\circ}$, and derived the expression for shear stiffness, $\mathrm{G}_{x y}$.

\subsection{Finite Element Simulation}

A smooth muscle tissue strip consisting of cells embedded in a tissue matrix, is assumed to be a cylinder of length $1 \mathrm{~mm}$ and a radius of $0.175 \mathrm{~mm}$. We further assume that it contains a single cylindrical cell of length $0.5 \mathrm{~mm}$ and radius $0.07 \mathrm{~mm}$ aligned at an off-axis orientation $\theta$ to the major axis. This smooth muscle cell-tissue strip is modeled as 3D solid with SOLID-175 finite elements using ANSYS-6.1 commercial software [9]. The final finite element model of smooth muscle cell-tissue cylinder consisted of 16482 SOLID-175 elements and 23828 nodes.

The material properties for the finite element model were assumed to be isotropic for both tissue and cell. The Young's modulus values are in the range $(\mathrm{E}=1 \times$ $10^{4}$ to $1 \times 10^{7}$ ) for cell and tissue. Further, a value of 0.44 for Poisson ratio was used for the material. The boundary conditions imposed were similar to those used in medical experiments, i.e., the smooth muscle is clamped at one end and at the other end, a constant compressive force (in the form of uniform displacement is distributed over the cross-section). Several structural analysis runs were carried out to study the effect of Von-mises stress for various displacements and off-axis cell orientations. The results are discussed in detail in the next section. 


\section{RESULTS AND DISCUSSION}

Shear stiffness $\mathrm{G}_{x y}(\theta)$ is estimated using $\mathbf{E q . 2}$ and the derived results from the experimental data for the on-axis stiffness $\mathrm{E}_{x x}\left(\theta=0^{\circ}\right)$. Figure 2 depicts the behavior of shear stiffness as a function of shortening strain and off-axis cell orientation $(\theta)$. It can be seen from Figure 2 that the shear stiffness values decreased with increasing shortening strain, and this decrease in shear stiffness values increased with higher off-axis cell orientations. For example, there was a $120 \%$ shear stiffness reduction for $20 \%$ shortening when the cells were oriented at $30^{\circ}$. But at lower off-axis cell orientations, the stiffness reduction was much less. Also, the initial shear stiffness values for $19.5^{\circ}$ and $30^{\circ}$ off-axis cell orientations was about 2.2 and 4.7 times higher than those of the stiffness values at $11^{\circ}$ off-axis cell orientation. There was a non-linear behavior of shear stiffness with off-axis orientation and this non-linear behavior increased with higher off-axis cell orientation.

Figure 3 shows the deformation of the cell oriented at $30^{\circ}$ under shortening strains of $5 \%, 35 \%$ and $50 \%$, respectively. It can be seen from Figure 3 that the cell deformation as well as the initial cell orientation changes as the shortening strain increases. It has been observed that the cell reorients by about two times the initial orientation under $50 \%$ shortening. Figure 4 shows the cut section views of undeformed and deformed cell-tissue model for a cell oriented at $30^{\circ}$ under shortening strain of $45 \%$. It can be seen from Figure 4 that once again a substantial amount of cell reorientation takes place. It should be noted that the change in initial cell orientation with shortening strain should be considered for a realistic analysis in order to see how it affects the shear stresses.

The deformed shapes of the cell for various shortening strains were determined as a function of off-axis angles. Figure 5 shows the results of both undeformed and deformed cells oriented at $0^{\circ}, 30^{\circ}$, and $45^{\circ}$ under shortening strains of $20 \%$ (Figure 5 left) and $45 \%$ (Figure 5 right). From the results of Von-Mises stresses presented in Figure 5, it can be seen that, both the deformation and stresses increase with increasing cell orientation and also with increasing strain. These results indicate that, in general, the cell is displaced from its initial position with angle shift depending on the strain applied and off-axis angle orientation $\theta$.

The effect of off-axis cell orientation and shortening strain on maximum shear stress is shown in Figure 6. It is evident from Figure 6, that the maximum shear stress values of the tissue at $45 \%$ strain are 2.5 times the corresponding values at $20 \%$ strain for off-axis orientation values $\theta=15^{\circ}, 30^{\circ}$ and $45^{\circ}$. In general, the results presented in Figures 5 and $\mathbf{6}$ illustrate the effect of cell orientation and shortening strains on shear stresses and deformation.

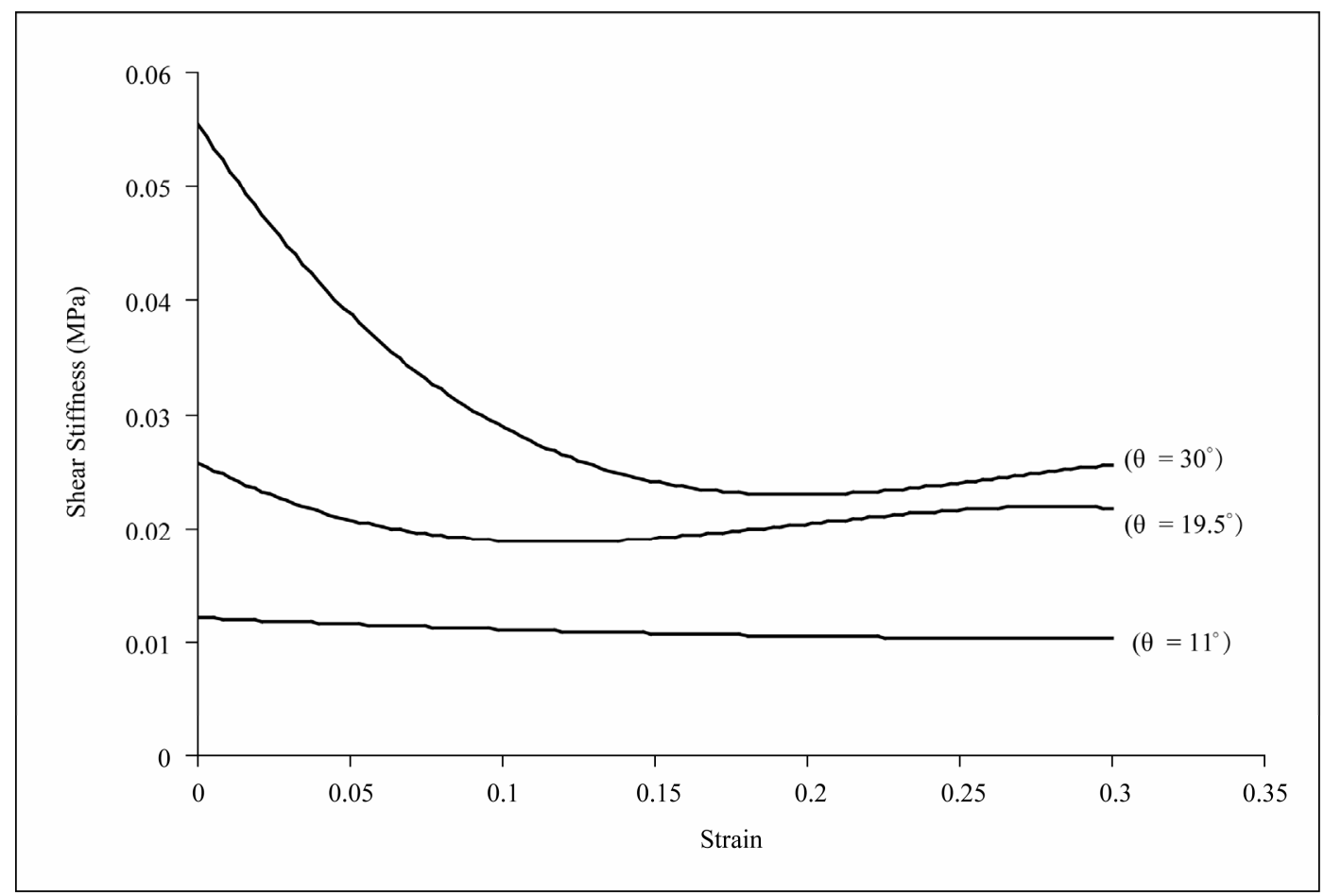

Figure 2. Shear stiffness-strain behavior predicted for off-axis cell orientations of $\theta=11^{\circ}, 19.5^{\circ}$ and $30^{\circ}$ in a smooth muscle tissue. 

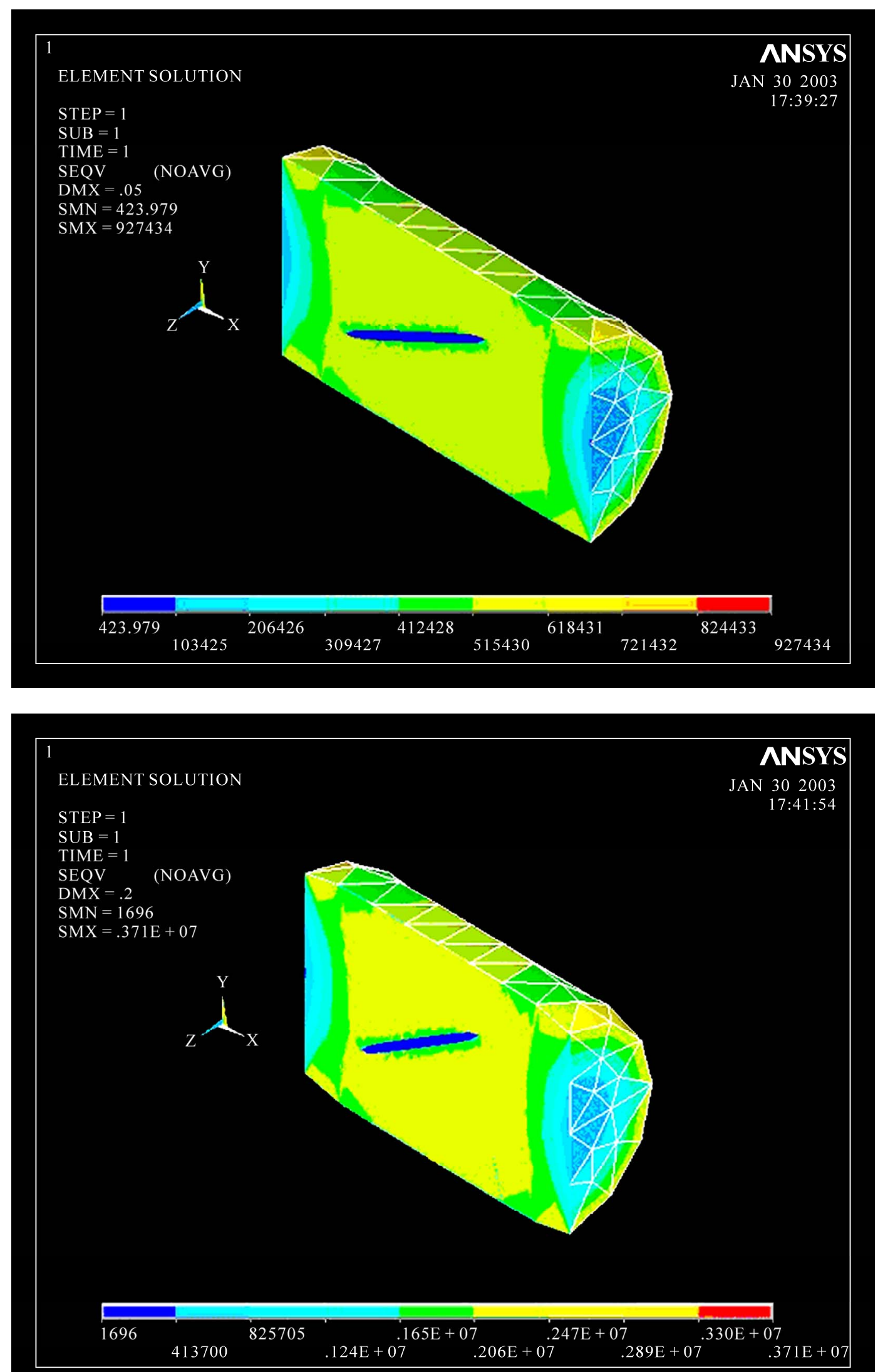


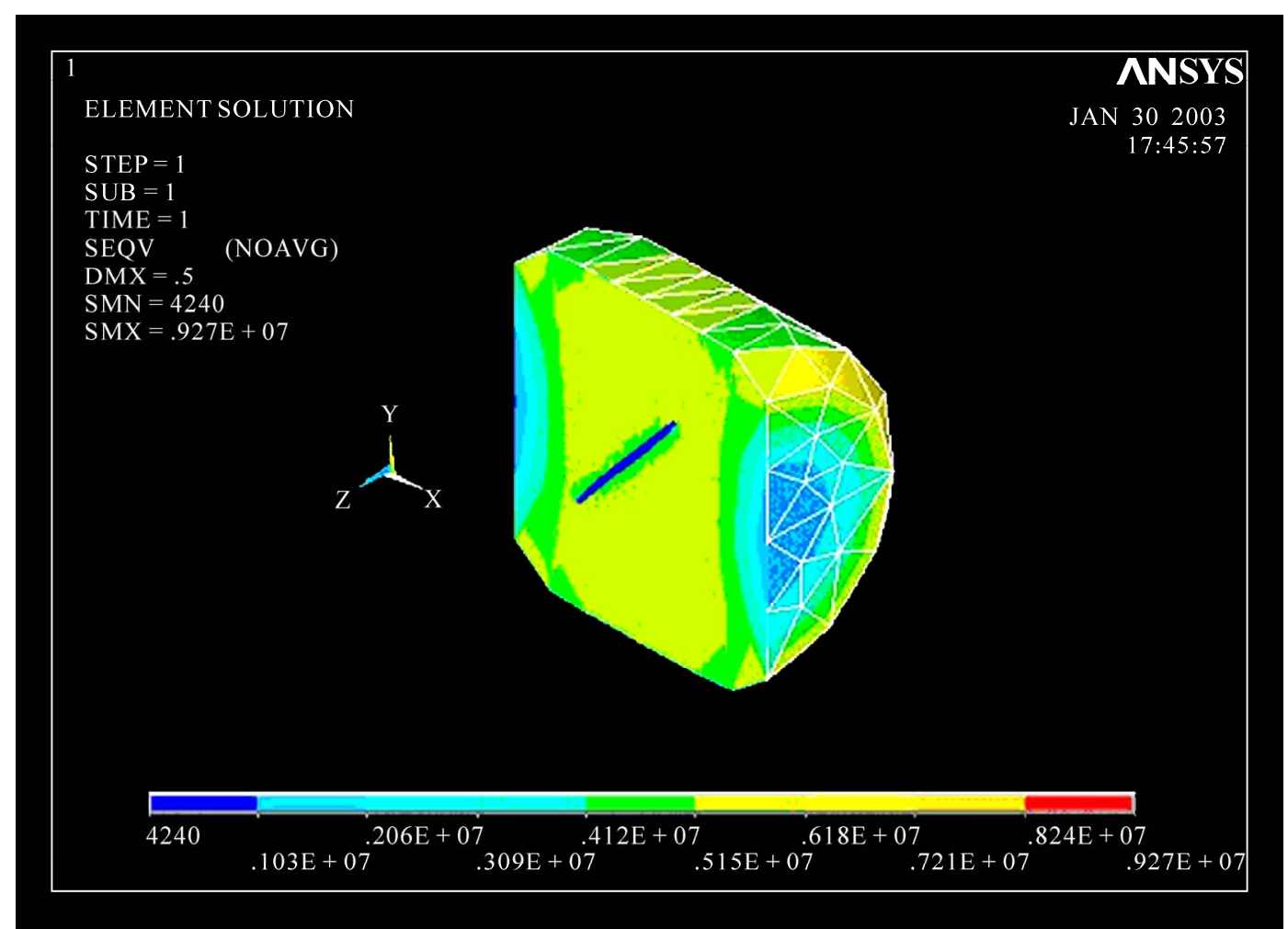

Figure 3. Cut section view of the deformation for a cell oriented at $30^{\circ}$ under shortening strains of 5\%, 35\% and $50 \%$.

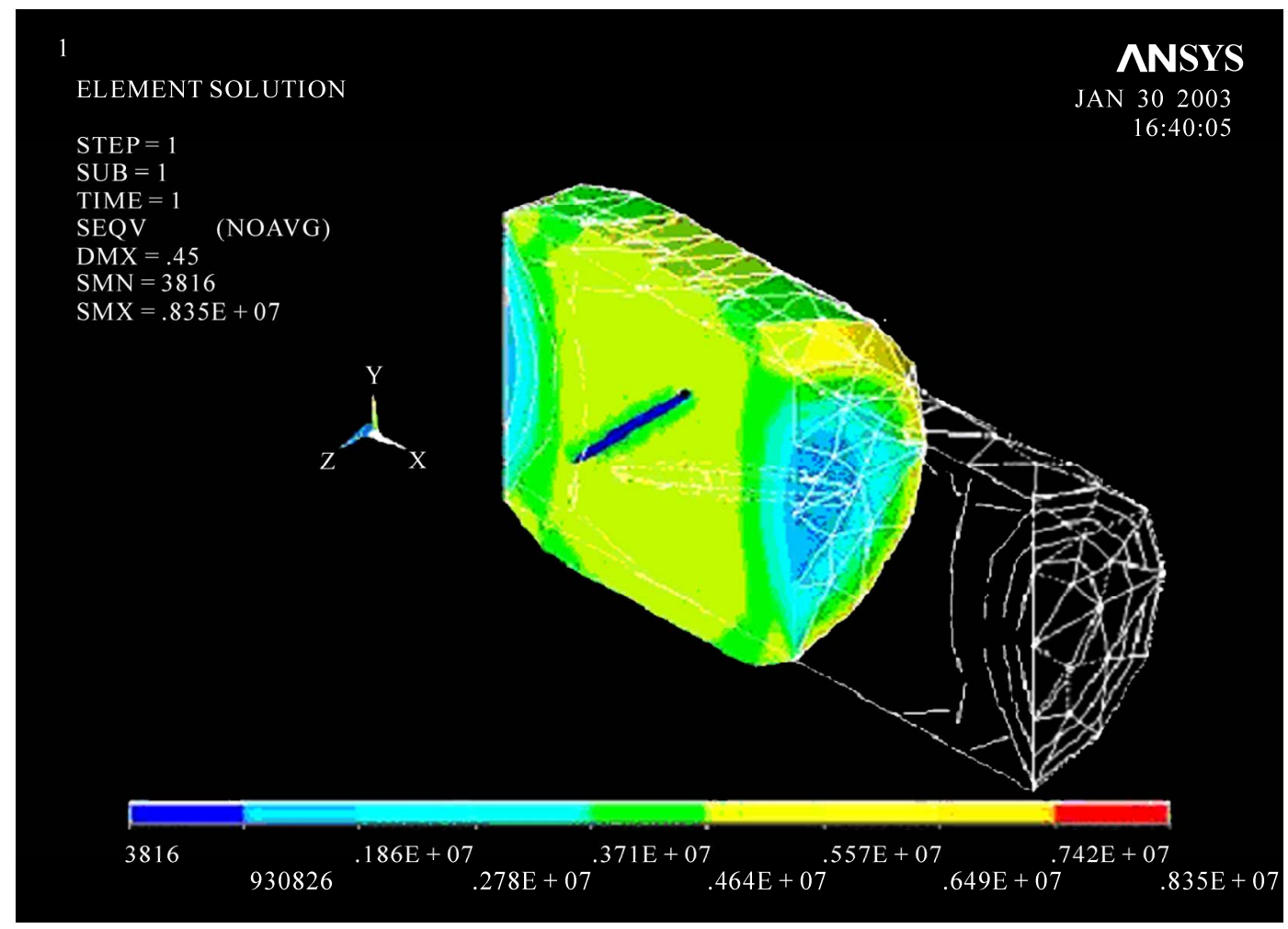

Figure 4. Cut section view of the undeformed and deformed cell-tissue model for a cell oriented at $30^{\circ}$ under shortening strain of $45 \%$. 

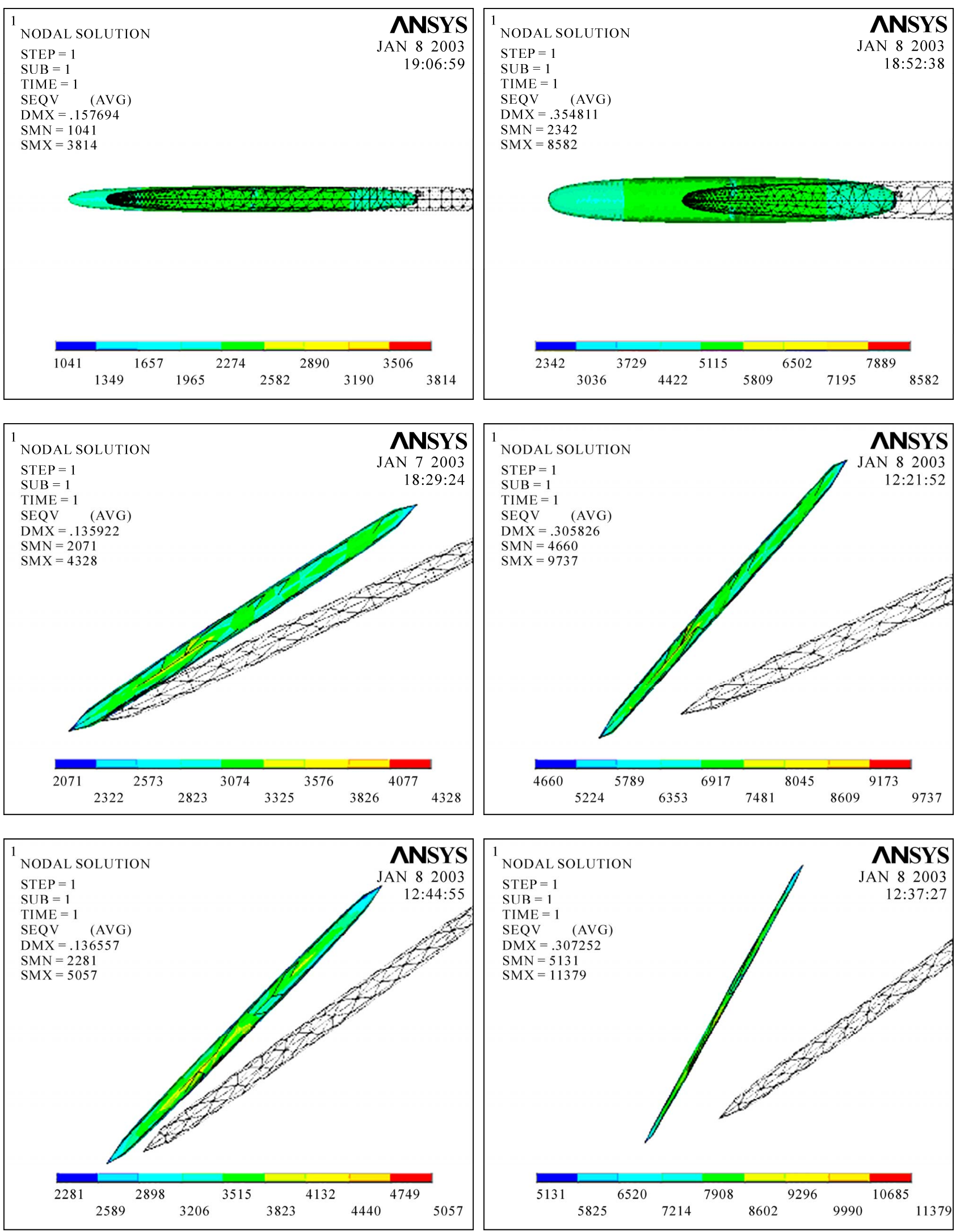

Figure 5. Deformed and undeformed cell shapes along with Von-Mises stresses at strains of 20\% (left) and $45 \%$ (right). 


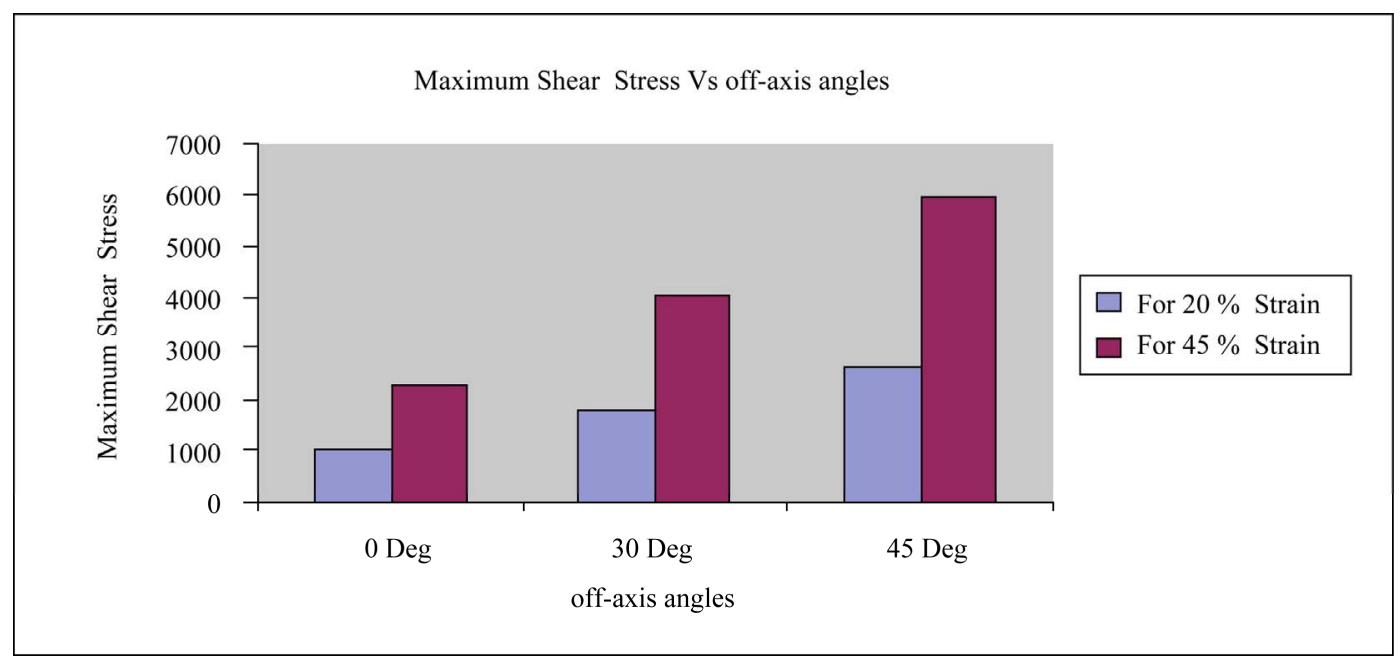

Figure 6. Effect of off-axis cell orientation and shortening strain on maximum shear stress.

\section{CONCLUSIONS}

This study investigated how the alignment of smooth muscle cells affects the shear stiffness of a tracheal smooth muscle strip through a simple model derived from experiments. Also, finite element analysis was carried out to study the effect of off-axis cell orientation on deformation and shear stresses. Shear stiffness results obtained from the model indicate that the muscle shear stiffness values increase non-linearly with increasing value of strain and with higher off-axis alignment of cells. One limitation of the model is that it does not take into account interactions between active and passive tissue components. Results of Von-mises stresses obtained indicate that the maximum shear stress values of tracheal smooth muscle increase with increasing values of shortening strain for each off-axis cell orientation.

\section{ACKNOWLEDGEMENTS}

The authors thank the U. S. National Science Foundation for supporting this work through a grant IBN-9904610.

\section{REFERENCES}

[1] Seow, C.Y. and Stephens, N.L. (1989) Changes of tracheal smooth muscle stiffness during an isotonic contrac- tion. American Journal of Physiology, 256, C341-C350.

[2] Meiss, R.A. (1999) Influence of intercellular tissue connections on airway muscle mechanics. Journal of Applied Physiology, 86, 5-15.

[3] Meiss, R.A. (2001) Shortening-dependent stiffness of tracheal smooth muscle is independent of temperature. Journal of Biophysics, 80, 274a.

[4] Sarma, P.A., Pidaparti, R.M. and Meiss, R.A. (2003) Anistropic properties of tracheal smooth muscle tissue. Journal of Biomedical Materials Research, 65, 1-18. doi:10.1002/jbm.a.10355

[5] Meiss, R.A. and Pidaparti, R.M. (2002) Mechanical effects of off-axis cell orientation in a smooth muscle strip. Biophysical Journal, 82, 371a.

[6] Meiss, R.A. and Pidaparti, R.M. (2003) Altered cellular alignment affects shortening in a smooth muscle strip. Biophysical Journal, 84, 104a.

[7] Pidaparti, R.M.V., Chandran, A., Takano, Y. and Turner, C.H. (1996) Bone mineral lies mainly outside collagen fibrils: Predictions of a composite model for osteonal bone. Journal of Biomechanics, 29, 909-916. doi:10.1016/0021-9290(95)00147-6

[8] Jones, R.M. (1975) Mechanics of composite materials. McGraw-Hill, New York.

[9] ANSYS 6.1 (2001) Finite element software. Pittsburgh, PA. 